\title{
Microstructural Stability of the Kirkendall Plane in Solid-State Diffusion
}

\author{
M. J.H. van Dal, A. M. Gusak,* C. Cserháti, A. A. Kodentsov, and F. J. J. van Loo ${ }^{\dagger}$ \\ Laboratory of Solid State and Materials Chemistry, Einghoven University of Technology, P.O. Box 513, \\ 5600 MB Eindhoven, The Netherlands
}

(Received 3 November 2000)

\begin{abstract}
In a diffusion-controlled interaction, the Kirkendall plane, as marked by inert particles placed at the original contact surface of a reaction couple, need not be unique. Multiple planes can develop, and sometimes the Kirkendall plane does not exist at all. A phenomenological approach is introduced to rationalize the Kirkendall-effect-mediated migration of macroscopic inclusions inside a diffusion zone.
\end{abstract}

PACS numbers: 66.30.-h

In 1947, Ernest Kirkendall reported the results of his study on the interdiffusion between $\mathrm{Cu}$ and $\alpha(\mathrm{Cu}, \mathrm{Zn})$ brass [1]. In his experiments he marked the contact interface between $\mathrm{Cu}$ and brass, prior to annealing, with inert thin molybdenum wires, and observed that this planar array of wires (marker plane, nowadays called the Kirkendall plane) shifted with respect to the ends of the diffusion couple. This phenomenon has been known since then as the Kirkendall effect. It played a decisive role in the development of the solid-state diffusion theory, as it is seen as the most explicit evidence for the occurrence of a vacancy mechanism in most solid-state diffusion processes. In fact, in a binary system it shows the different independent intrinsic diffusion fluxes of the components $A$ and $B$ [2], which causes swelling of one part and shrinking of the other part of the couple, leading to the marker displacement.

The reason for writing this Letter is the need for a reexamination of some basic concepts involved in the analysis of the marker behavior. We do not go into the atomic mechanisms of diffusion, but we will concentrate on the phenomenological description of the Kirkendall effect. Especially significant is the experimental fact that the Kirkendall plane stays at the same composition during a diffusion-controlled process, moving parabolically in time with a velocity $v=\left(x_{K}-x_{0}\right) / 2 t$. Here $x_{K}$ is the position of the Kirkendall markers at annealing time $t$ and $x_{0}$ is their position at time $t=0 ; x_{K}-x_{0}$ is, therefore, the displacement of the markers.

The most important conclusion, emerging from our experimental studies over the last years [3-5], is that in a volume-diffusion controlled interaction the Kirkendall plane need not be unique as was tacitly assumed up to now. The experimental evidence was found by using as inert markers very fine agglomerates of $\mathrm{ThO}_{2}$ particles, $0.5-5 \mu \mathrm{m}$ in size, which were evenly spread at the contact surface of the couple in such a way that the interdiffusion was not hindered and each particle can move individually (unlike wires that are 10-25 $\mu \mathrm{m}$ in thickness and move as a whole). In some cases we found $\mathrm{ThO}_{2}$ particles back after annealing in two distinct rows, each moving with a (different) velocity $v=x / 2 t$. In other cases we found the
$\mathrm{ThO}_{2}$ particles back not as a straight row, but dispersed in a part of the diffusion couple. We will discuss some examples and rationalize the Kirkendall effect in a new approach that takes care of all experimental findings.

The formation of two "Kirkendall planes" moving with different velocities was, for instance, observed in a layer of $\beta^{\prime}$-AuZn (B2; CsCl-structure), diffusion grown during solid-state reaction at $500{ }^{\circ} \mathrm{C}$ between $\mathrm{Au}$ and $\gamma-\mathrm{AuZn}_{2}$ (Fig. 1). The Kirkendall planes are revealed by the straight rows of $\mathrm{ThO}_{2}$ particles used as markers between the initial end members. We note that at this temperature the $\beta^{\prime}$ phase exhibits a wide compositional range (38.5-56.0 at. \% of $\mathrm{Zn} \mathrm{[6])} \mathrm{and} \mathrm{that} \mathrm{in} \mathrm{the} \mathrm{Au-rich} \mathrm{part} \mathrm{of}$ the homogeneity region $\mathrm{Au}$ is the faster diffusing species, whereas in the Zn-rich part, Zn-atoms diffuse faster [7].



FIG. 1. Back-scattered electron image (BEI) of a $\mathrm{Au} /$ $\mathrm{Au}_{36} \mathrm{Zn}_{64}$ (" $\gamma-\mathrm{AuZn}{ }_{2}$ ") diffusion couple annealed at $500{ }^{\circ} \mathrm{C}$ for $17.25 \mathrm{~h}$ under flowing argon. After interdiffusion the $\mathrm{ThO}_{2}$ markers introduced between the couple halves are clearly visible as two distinct straight rows of inclusions. 
The appearance of more than one Kirkendall plane is not restricted to interdiffusion zones composed of a singlephased reaction product. For instance, Fig. 2(a) shows a multiphase reaction zone developed in a $\mathrm{Ti} / \mathrm{Ni}$ diffusion couple annealed in vacuum at $850{ }^{\circ} \mathrm{C}$ for $196 \mathrm{~h}$. Three intermetallics, $\mathrm{TiNi}_{3}, \mathrm{TiNi}$, and $\mathrm{Ti}_{2} \mathrm{Ni}$, are formed. Before annealing, $\mathrm{ThO}_{2}$ particles were introduced between the end-members to mark the initial contact surface. After interaction, two Kirkendall planes were clearly observed within the zone of interdiffusion.



(a)

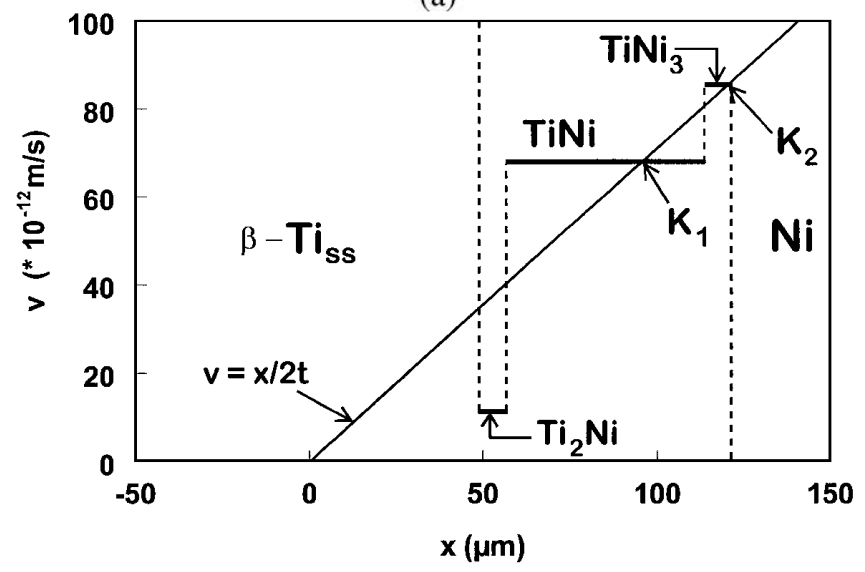

(b)

FIG. 2. (a) BEI of a cross section of a $\mathrm{Ti} / \mathrm{Ni}$ diffusion couple annealed at $850{ }^{\circ} \mathrm{C}$ for $196 \mathrm{~h}$. $\mathrm{ThO}_{2}$ particles were used as inert markers between the initial end members (the $\beta-\mathrm{Ti}_{\mathrm{ss}}$ solid solution layer is very thick and the $\alpha$-Ti end member is far away from the intermetallic layers shown in the picture); (b) The computed Kirkendall velocity plot. Note: $x=0$ corresponds to the Matano plane, the location of the initial contact surface at $t=0$. The Kirkendall velocity in the $\beta-\mathrm{Ti}_{\mathrm{ss}}$ phase was estimated as $10^{-9} \mathrm{~m} / \mathrm{s}$.
The migration of macroscopic inert inclusions within the zone of interdiffusion can be rationalized in terms of a so-called Kirkendall velocity curve [3,8]. This plot displays the velocities of inert particles, placed prior to the annealing at various locations within the anticipated interaction zone, relative to the couple end. In a binary system the Kirkendall velocity $v$ is dependent on the difference in intrinsic diffusivities of the species and can be calculated taking into account the volume changes accompanying interdiffusion [9]:

$$
\begin{aligned}
& v=-\left(V_{A} J_{A}+V_{B} J_{B}\right), \\
& v=\frac{V_{A} V_{B}}{V_{m}^{2}}\left(D_{A}-D_{B}\right) \frac{\partial N_{A}}{\partial x} .
\end{aligned}
$$

$D_{i}$ is the intrinsic diffusion coefficient of component $i$ $\left(\mathrm{m}^{2} / \mathrm{s}\right)$, defined by $J_{i}=-D_{i}\left(\partial C_{i} / \partial x\right)$ with $J_{i}$ being the intrinsic flux with respect to the Kirkendall frame of reference, and $C_{i}$ being the concentration of component $i$ (mole of atoms $/ \mathrm{m}^{3}$ ). $V_{i}$ is the partial molar volume of component $i\left(\mathrm{~m}^{3} /\right.$ mole of atoms), $V_{m}$ is the molar volume ( $\mathrm{m}^{3} /$ mole of atoms), $N_{A}$ is the mole fraction of $A$, and $x$ is the position parameter $(m)$.

The Kirkendall velocity curve can also be measured experimentally using, e.g., the "multifoil" method $[3,10]$.

As mentioned above, the particles placed at the Kirkendall plane (original contact surface) are the only markers which move parabolically in time with a velocity $v=$ $x / 2 t$. Obviously, the location (and velocity) of the Kirkendall plane after interdiffusion time $t$ can be determined from the intersection of the velocity curve and the straight line $v=x / 2 t$.

As an illustration of the approach, let us consider an example of marker behavior in the multiphase $\mathrm{Ti} / \mathrm{Ni}$ diffusion couple discussed above. For the sake of simplicity, it is assumed that no volume change occurs during the interaction and the binary Ti-Ni phases are treated as line compounds. In such a case, the Kirkendall velocity in any phase can be written as $[3,4]$

$$
v=\frac{\left(D_{A} / D_{B}\right)-1}{\left(D_{A} / D_{B}\right) N_{B}+N_{A}}\left(\frac{\tilde{D}_{\text {int }}}{\Delta x}\right),
$$

where $\tilde{D}_{\text {int }}$ is the integrated diffusion coefficient $\left(\mathrm{m}^{2} / \mathrm{s}\right)$ in the phase $[9,11]$ and $\Delta x$ is the layer thickness.

The velocity plot corresponding to the $\mathrm{Ti} / \mathrm{Ni}$ diffusion couple is shown in Fig. 2(b). The position $x=0$ is the location of the original contact surface. One can see that the straight line $v=x / 2 t$ intersects the Kirkendall velocity curve twice. This implies that two "Kirkendall planes" are expected inside the interaction zone, one in the TiNi and one in the $\mathrm{TiNi}_{3}$ phase. Indeed, two planes marked by $\mathrm{ThO}_{2}$ particles originally introduced at the contact $\mathrm{Ti} / \mathrm{Ni}$ interface were experimentally found within the predicted intermetallics in this diffusion couple [Fig. 2(a)]. Similar behavior of inert markers has been observed in the binary Co-Si system [5]. 
Another important observation relevant to the present discussion concerns the stability (spatial and temporal) of the Kirkendall plane as marked by fiducial particles. This can be best explained by an example of a hypothetical $A / B$ diffusion couple with a growing layer of a nonstoichiometric binary compound $\gamma$, in which on the $A$-rich side $A$ is the fastest diffusing species and on the $B$-rich side $B$ diffuses faster. (Note: This situation parallels that in the $\beta^{\prime}$-AuZn phase layer during interdiffusion in the $\mathrm{Au} / \gamma$ $\mathrm{AuZn}_{2}$ couple discussed previously.)

In Fig. 3, the corresponding velocity curve is given schematically. It is possible for the line $v=x / 2 t$ to intersect the Kirkendall velocity curve up to three times as is shown in the figure. This implies that in such a diffusion couple three Kirkendall planes might exist, which move parabolically in time. As a result, the markers originally introduced between the end members would rearrange during interdiffusion into three distinct parallel rows of inclusions, situated at the positions $K_{1}, K_{2}$, and $K_{3}$. However, one should realize that only two of the three predicted Kirkendall planes can be found experimentally within the reaction zone. This can be appreciated by analyzing the stability of the Kirkendall plane at the possible intersections. If markers, which ended up at the position $K_{1}$ in Fig. 3, would (for whatever perturbation) be slightly ahead of this plane, they would slow down (lower velocity), and if these markers were behind this plane, they would move faster (higher velocity). In other words, the plane located at $K_{1}$ tends to "attract" inert markers in its vicinity. Hence, a stable Kirkendall plane will emerge in the interdiffusion zone when the straight-line $v=x / 2 t$ intersects the velocity curve at a position where the Kirkendall velocity has a negative gradient.

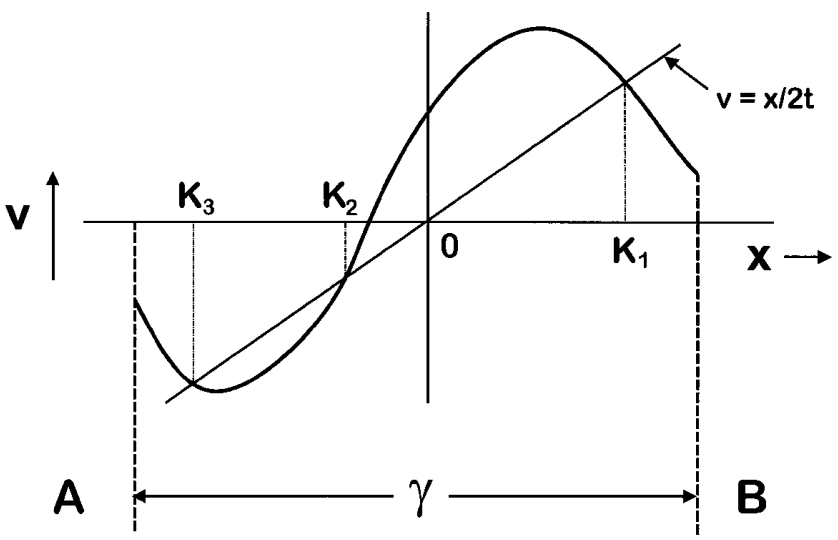

FIG. 3. The Kirkendall velocity in a layer of a nonstoichiometric $\gamma$ phase growing in a hypothetical $A-B$ diffusion system (schematically). The intrinsic diffusion coefficients of $A$ and $B$ in $\gamma$ are chosen arbitrarily in such a way that on the $A$ side of the diffusion zone, $A$ is the faster diffusing species and on the $B$ side, the component $B$ has the highest diffusivity. (The position $x=0$ corresponds to the Matano plane). Three intersections $K_{1}, K_{2}$, and $K_{3}$ are found, of which only two correspond to stable Kirkendall planes $\left(K_{1}\right.$ and $\left.K_{3}\right)$.
Following a similar line of argument, it can be concluded that an unstable Kirkendall plane will appear inside a diffusion zone when the gradient of the velocity is positive at the intersection point. Indeed, the markers which are slightly ahead of the plane situated at $K_{2}$ in Fig. 3 will move faster,

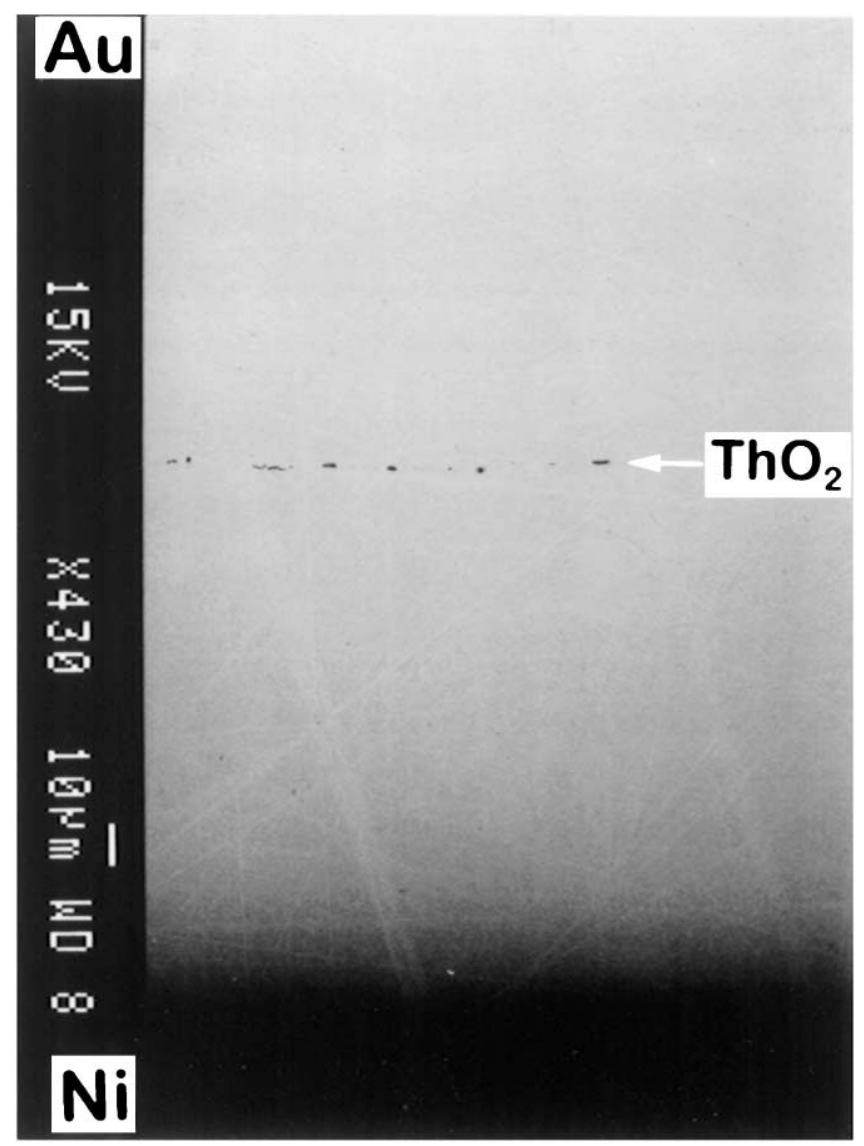

(a)

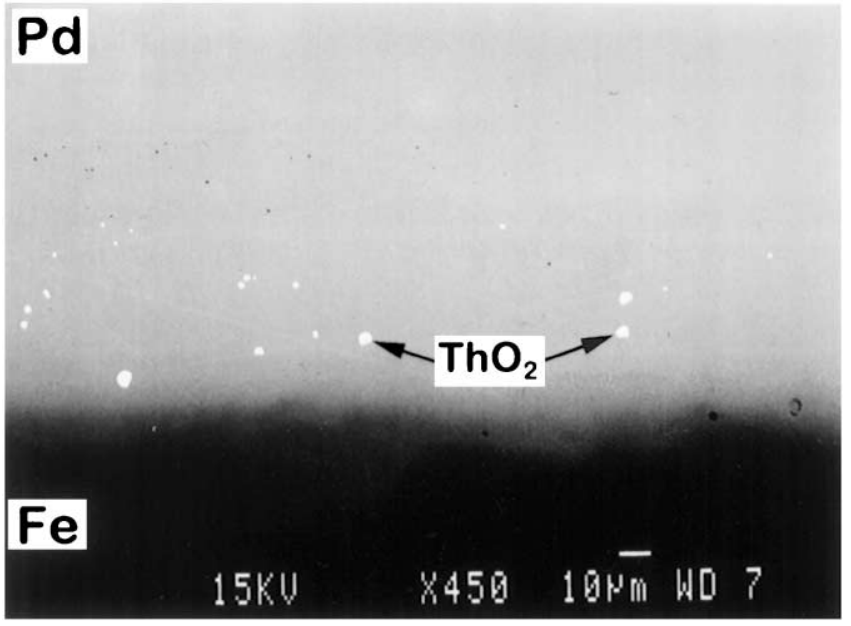

(b)

FIG. 4. BEI of (a) a Au/Ni diffusion couple annealed at $900{ }^{\circ} \mathrm{C}$ in vacuum for $50 \mathrm{~h}$, and (b) an $\mathrm{Fe} / \mathrm{Pd}$ couple after interdiffusion at $1100{ }^{\circ} \mathrm{C}$ in vacuum for $144 \mathrm{~h}$. The $\mathrm{ThO}_{2}$ markers exhibit a dark-gray contrast on the $\mathrm{BEI}$ image of the $\mathrm{Au} / \mathrm{Ni}$ diffusion zone and a white contrast in the case of the $\mathrm{Fe} / \mathrm{Pd}$ couple. 

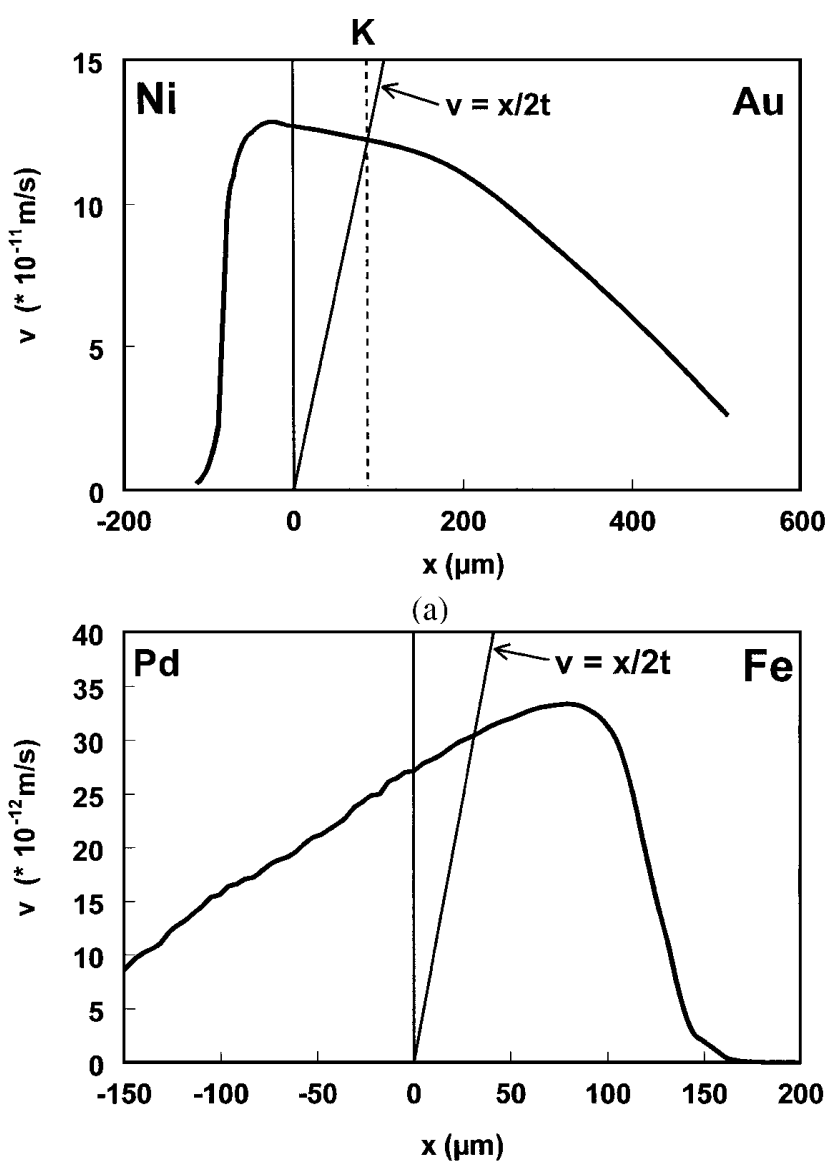

(b)

FIG. 5. Experimentally determined Kirkendall velocity curves of (a) a multifoil $\mathrm{Au} / \mathrm{Ni}$ couple annealed at $900{ }^{\circ} \mathrm{C}$ for $100 \mathrm{~h}$ and (b) a multifoil $\mathrm{Fe} / \mathrm{Pd}$ couple annealed at $1100{ }^{\circ} \mathrm{C}$ for $144 \mathrm{~h}$.

and markers slightly behind this plane will migrate slower. If such an unstable Kirkendall plane is situated between two stable ones (as in the example in Fig. 3), the stable planes will accumulate all markers during the initial stage of interdiffusion. Therefore, only two Kirkendall planes (at the positions $K_{1}$ and $K_{3}$ ) are expected to appear in the $A / B$ couple as found, indeed, in the Au-Zn system shown in Fig. 1.

A particularly convincing example of the formation of stable and unstable Kirkendall planes is provided by the observations made in the study on the Kirkendall effect in $\mathrm{Au}-\mathrm{Ni}$ and $\mathrm{Fe}-\mathrm{Pd}$ solid solution systems. Figure 4 shows back-scattered electron images of a $\mathrm{Au} / \mathrm{Ni}$ and an $\mathrm{Fe} / \mathrm{Pd}$ diffusion couple annealed at 900 and $1100{ }^{\circ} \mathrm{C}$, respectively.

One can see a remarkable difference in the appearance of the markers inside the interdiffusion zones. In the $\mathrm{Au}-\mathrm{Ni}$ system, the $\mathrm{ThO}_{2}$ particles form a planar array of platelike inclusions within the diffusion zone. In the $\mathrm{Fe} / \mathrm{Pd}$ couple, on the other hand, a significant scattering of the particles occurred upon interaction.

The Kirkendall velocity curves corresponding to the couples were constructed experimentally using the multifoil diffusion couple technique [10] (Fig. 5). More details concerning this method can be found elsewhere [3].

In both cases, the line $v=x / 2 t$ intersects the velocity curve once. In the $\mathrm{Au} / \mathrm{Ni}$ diffusion couple, the velocity curve at the intersection point has a negative slope, which results in the formation of a single, stable Kirkendall plane inside the interdiffusion zone [Fig. 5(a)]. The appearance of the marker array as a straight row of platelike $\mathrm{ThO}_{2}$ particles reflects the tendency of a stable Kirkendall plane to accumulate the markers in its vicinity. By contrast, in the case of the $\mathrm{Fe} / \mathrm{Pd}$ couple, the straight line intersects the velocity curve at a point where the Kirkendall velocity has a positive gradient, which will lead to an unstable Kirkendall plane [Fig. 5(b)]. The initial planar array of the markers at the contact interface tends to transform into an array of $\mathrm{ThO}_{2}$ particles spatially distributed in the diffusion direction. One could say that no Kirkendall plane in the $\mathrm{Fe} / \mathrm{Pd}$ diffusion couple exists at all.

We would like to thank Professor A.M. Glaeser from the University of California, Berkeley, for many helpful discussions.

*On leave from Cherkasy State University, Ukraine.

${ }^{\dagger}$ To whom all correspondence should be addressed. Email address: F.J.J.v.Loo@tue.nl

[1] A. D. Smigelskas and E. O. Kirkendall, Trans. AIME 171, 130 (1947).

[2] L. S. Darken, Trans. AIME 175, 184 (1948).

[3] M. J. H. van Dal, M. C. L. P. Pleumeekers, A. A. Kodentsov, and F. J. J. van Loo, Acta Mater. 48, 385 (2000).

[4] M. J. H. van Dal, M. C. L. P. Pleumeekers, A. A. Kodentsov, and F. J. J. van Loo, J. Alloys Compd. 309, 132 (2000).

[5] M. J.H. van Dal, A. A. Kodentsov, and F. J. J. van Loo, Solid State Phenom. 72, 111 (2000).

[6] H. Okamoto and T. B. Massalski, Phase Diagrams of Binary Gold Alloys (ASM International, Metals Park, Ohio, 1988).

[7] D. Gupta and D. S. Lieberman, Phys. Rev. B 4, 1070 (1971).

[8] J.-F. Cornet and D. Calais, J. Phys. Chem. Solids 33, 1675 (1972).

[9] F. J. J. van Loo, Prog. Solid State Chem. 20, 47 (1990).

[10] Th. Heumann and G. Walther, Z. Metallkd. 48, 151 (1957).

[11] C. Wagner, Acta Metall. 17, 99 (1969). 